\title{
LA INTRODUCCIÓN DE VARIEDADES DE TRIGO DURANTE EL PROCESO DE CONSTITUCIÓN DEL MODELO AGRÍCOLA ESTADOUNIDENSE
}

Francisco Javier

\section{SERRANO-BOSQUET}

\author{
(D) fjavierserrano@tec.mx
}

Tecnologico de Monterrey

Monterrey, México

The introduction of wheat varieties during the constitution process

of the american agricultural model

\begin{abstract}
The main objective of this paper is to show the role played by the selection and creation of new wheat varieties in the process of constitution and territorial expansion of the US wheat production model from the 17th century until the end of the 19th century. The article suggests that these biological innovations allowed to increase the production and territorial extension of the wheat, they were key in the development of a commercial production on a large scale and contributed key empirical and methodological data for the constitution and institutionalization of a scientific agriculture at the beginning of the 20th century. These innovations were the result of very different efforts and experiences, from those personal, local or community accumulated over generations, to those based on the scientific, mathematical and methodological literature of the time.
\end{abstract}

Keywords: standardization, standardization, scientific agriculture, wheat, varieties, hybrids, history of agriculture.

\section{Resumen}

El objetivo principal de este trabajo es mostrar el papel que jugaron la selección y creación de nuevas variedades de trigo en el proceso de constitución y expansión territorial del modelo de producción de trigo estadounidense desde el siglo XVII hasta finales del XIX. El artículo sugiere que estas innovaciones biológicas permitieron aumentar la producción y extensión territorial triguera, fueron claves en el desarrollo de una producción comercial a gran escala y aportaron datos empíricos y metodológicos claves para la constitución e institucionalización de una agricultura científica a inicios del siglo XX. Dichas innovaciones fueron resultado de esfuerzos y experiencias muy dispares, desde aquellos personales, locales o comunitarios acumulados durante generaciones, hasta los basados en la literatura, matemática y metodológica científica de la epoca.

Palabras clave: estandarización, normalización, agricultura científica, trigo, variedades, híbridos, historia de la agricultura. 
I extraordinario aumento en la producción de trigo que disfrutó Estados Unidos durante el siglo XIX ha sido ampliamente estudiado. La mayor parte de la investigación se ha centrado en los aspectos económicos o la introducción de maquinaria cada vez más compleja adaptada a la producción agrícola. Sin embargo, en los últimos años hemos sido testigos de una proliferación de estudios e investigaciones que intentan resaltar el extraordinario papel desempeñado por las innovaciones biológicas ${ }^{1}$. El presente trabajo se suma a esta tendencia centrando su atención en el papel que desempeñaron -junto a otros tipos de esfuerzos- la selección y creación de nuevas variedades de trigo en el desarrollo y expansión territorial de la producción triguera estadounidense desde el siglo XVII hasta finales del siglo XIX .

Para ello, nos basamos en el análisis de fuentes primarias de finales del siglo XIX e inicios del XX, la revisión de artículos y libros del primer tercio del siglo XX y obras de referencia más actuales. El estudio de esta literatura, bajo el enfoque señalado, presenta el proceso de sistematización y estandarización de la producción agrícola como un desarrollo continuo, si bien cargado de choques, rivalides y distintas formas de entender y vivir el significado de la producción agrícola. Frente a la imagen común de una agricultura que podríamos llamar paleo o proto-estandarizada, resultado de experiencias personales, locales o comunitarias acumuladas a través de generaciones en granjas y establos, sin un soporte científico o metodológico, el artículo sugiere que muchas de las innovaciones biológicas desarrolladas en este periodo, mismas que permitieron aumentar la producción y facilitar la extensión territorial triguera, estuvieron basadas en la literatura, la matemática y las metodologías científicas de la epoca.

Llevamos a cabo, por consiguiente, un análisis histórico en el que hemos distinguido dos grandes periodos y tres regiones productivas. La primera etapa inicia con el siglo XVII y llega hasta la guerra civil estadounidense; la segunda, comienza con el periodo de postguerra y se extiende hasta las últimas décadas del siglo XIX. En ambos periodos, examinamos separadamente lo ocurrido en las costas este y oeste y sumamos, en el segundo periodo, los esfuerzos de expansión territorial llevados a cabo en las Grandes Llanuras Centrales. Poteriormente, analizamos el papel que desempeñó la introducción y el desarrollo de nuevas variedades de trigo en la construcción del modelo de producción de trigo norteamericano en un contexto de expansión comercial y territorial. Cerramos con una serie de análisis, reflexiones y conclusiones generales sobre papel desempeñado por la base teórica durante los períodos abordados durante el trabajo, así como el papel que jugó en la constitución del modelo productivo de trigo estadounidense del siglo $\mathrm{XX}$.

\section{Primeros pasos de la agricultura triguera estadounidense}

\section{La costa Este. La agricultura en Nueva Inglaterra²}

\section{Inicios y revolución agrícola en Nueva Inglaterra}

La principal característica distintiva de la vida en las granjas de Nueva Inglaterra fue, hasta inicios del siglo XIX, su autosuficiencia. Tan sólo producciones como la del tabaco tuvieron desde el inicio un claro perfil comercial ${ }^{3}$. Ello se debió, sobre todo, a 
las condiciones geográficas: las laderas escarpadas y los suelos rocosos y delgados, rara vez permitieron el desarrollo de unidades de cultivo grandes e intensas. Fueron, por tanto, las pequeñas fincas y propiedades familiares las que mejor se adaptaron inicialmente a este espacio (Schwartz 1995, 455). La ausencia de un importante mercado interno, y con ello la imposibilidad de vender sus productos y comprar aquellos otros que se necesitaban, obligó a las familias agrícolas a autoproveerse de todo lo que precisaran (Bidwell 1921, 684; True 1937, 1). Cada granja funcionaba como una unidad económica o microcosmos en la que se intentaba producir desde alimentos, ropa o muebles, hasta ropa de cama, jabón y velas (Bidwell 1921, 684). La forma en la que se distribuía la extensión de tierra dedicada a pastos, bosques y labranza, era práctivamente la misma en todas las granjas, se sembraban los mismos cultivos y se llevaban a cabo los mismos tipos de acciones. Durante las primeras décadas del siglo XIX dos fenómenos vendrían a poner en riesgo este modelo: un extraordinario aumento de la población urbana y la proliferación de vías férreas.

Entre 1810 y 1860 la población de Nueva Inglaterra creció alrededor de un 130\%, concentrandose la mayoría de ella en las zonas urbanas. Este aumento poblacional no agrícola fue de suma importancia para los agricultores, ya que hizo posible que, por primera vez, tuvieran un importante mercado regional al que dirigir sus productos (Bidwell 1921, 685). La aparición de este mercado estimuló el desarrollo de la agricultura comercial hasta el punto en que la transición de una agricultura de subsistencia a otra comercial necesitara de tan sólo dos generaciones ${ }^{4}$. Ahora bien, las consecuencias que hubo que enfrentar a raíz de los cambios generados, fueron mucho más allá de los relacionados con una simple transformación de los modelos de producción. Significaba, en primer lugar, poner en riesgo los valores de autosuficiencia, independencia, frugalidad y ahorro sobre los que se asentaba la agricultura tradicional 5; se ponía en riesgo además la participación de todos los miembros de la familia en las tareas de la granja (principalmente a las esposas e hijas) ${ }^{6}$ y la prevalencia de los intereses del grupo familiar sobre los intereses de sus miembros individuales. Obligaba a los agricultores, asímismo, a hacer frente a nuevos problemas y exigía desarrollar competencias para las que no estaban preparados: comprar maquinaria y fertilizantes, vender, administrar y gestionar sus productos a mediana o gran escala.

La construcción de numerosas vías ferreas en la década de 1840 tuvo dos importantes consecuencias que contribuyeron a aumentar la presión que ya entonces soportaban los agricultores yanquis. En primer lugar, permitió a los productores tener acceso a un gran mercado nacional; por otro, les expuso a una competencia para la que no estaban todavía preparados. Cierto es que los agricultures de Nueva Inglaterra nunca disfrutaron del monopolio de su mercado. Antes de 1810 tuvieron que competir con el comercio marítimo, lidiar con la apertura del Canal Erie y hacer frente a la competencia generada por la introducción de los barcos a vapor en Long Island Sound y el río Connecticut. Ahora bien, el establecimiento de nuevas conexiones con Occidente a través del ferrocarril entre 1840 y 1850 , vino a tensar aún más la situación, llegándo a convertirse en la principal fuente de estrés de los agricultores durante este periodo crítico (Bidwell 1921, 689). 
Ambos factores, la oportunidad de vender productos agrícolas en nuevos mercados y tener que hacer frente a la nueva competencia, estimuló un cambio que comenzó con la especialización (Bidwell 1921, 683). Sin embargo, hubo un obstáculo pocas veces señalado que frenó y condicionó el progreso de la agricultura en esta época. Se trata, tal y como señala Bidwell (1921, 700), del abandono de las fincas por parte de la generación más joven. Numerosos hijos de agricultores abandonaron sus hogares para buscar fortuna como oficinistas y obreros en las ciudades. Y, entre aquellos jóvenes que quisieron seguir la carrera agrícola, muchos prefirieron emigrar hacia el Oeste. La principal consecuencia fue que, justo en el momento en el que hacía más falta mano de obra barata y fiable para sacar el máximo provecho a las nuevas oportunidades de mercado, ésta desaparecía. Además, lo hizo en primer lugar el mejor material humano, los mejores cerebros y los espíritus más audaces (Bidwell 1921, 701).

De ahí que, si cierto es que un pequeño grupo de agricultores hizo posible que en tan pocas décadas pudiera hablarse ya de una agricultura comercial en Nueva Inglaterra, ésta no se consolidó hasta la posguerra civil. Hasta entonces, la mayoría de los agricultores continuaron haciendo las mismas cosas practicamente de la misma manera. ${ }^{8}$

Hasta aquí, hemos visto algunos elementos básicos de la primera etapa de la agricultura estadounidense, veamos ahora con algo más de detalle cómo se desarrolló durante la misma la producción triguera.

\section{Producción de trigo en Nueva Inglaterra}

La llegada de colonos de distintos países y comarcas europeas a lo largo de sucesivas migraciones, facilitó la introducción, prueba, adaptación y comparación de muchas variedades de trigo en un periodo de tiempo relativamente corto (Ball 1930, 51). Una introducción que comenzó muy pronto, ya que hay constancia de que fue sembrado por primera vez por Gosnold en Buzzards Bay en 1602, que se cultivaba en la colonia de Jamestown en 1611 y en Plymouth poco después de 1621. A partir de 1628 se introdujeron nuevas variedades inglesas en las nuevas colonias de Nueva Inglaterra y, a partir de 1634, en Maryland. El trigo holandés y sueco llegó con los colonos a Nueva York entre 1622 y 1626, a Nueva Jersey en 1622 y a Delaware en 1638, a las colonias inglesas de las Carolinas en 1663 y 1670 y, a Pennsylvania en 1682. Siguió introduciéndose trigos ingleses hasta el final del siglo XVII (Ball 1930, 51) ${ }^{9}$.

Si bien ni los suelos ni el clima de la mayor parte de la llanura costera atlántica eran propicios para el cultivo del trigo, los esfuerzos de mejora llevados a cabo durante este periodo fueron -contra lo que cabría esperar- muy pequeños ${ }^{10}$. De hecho, el cultivo del trigo languideció en las primeras colonias ya que el maíz se adaptaba mejor a las condiciones geoclimáticas noratlánticas, y su siembra y cosecha en condiciones pioneras con mano de obra e implementos primitivos, era más sencilla (Ball 1930, 51). 


\section{La costa Oeste. Los antecedentes novohispanos}

Encontramos más y más exitosos esfuerzos relacionados con la introducción, adaptación y cuidado de variedades de trigo en el otro extremo de los futuros Estados Unidos, en la costa del Pacífico. Se trata de aquellos programas agrícolas llevados a cabo en las regiones más septentrionales de Nueva España, en territorios que actualmente conforman los estados de Californiana, Arizona y Nevada."

Si bien no es momento este de entrar en una relación minuciosa de la historia de la producción triguera novohispana, sí es importante tener en cuenta que el origen de la producción triguera en los EEUU coincide en parte con los primeros pasos del cultivo de este cereal en el continente americano dados en el virreinato español.12 En ese sentido, es oportuno señalar que un hecho particularmente importante fue que en 1524 Hernán Cortés reglamentara "en las ordenanzas que dictó para los encomenderos de su jurisdicción la obligatoriedad de sembrar cereales españoles -trigo y cebada - en los pueblos de indios que tuviera cada uno a su cargo" (Del Río Moreno y López y Sebastián 1996, 34). Esta reglamentación fue clave en la rápida expansión del cultivo de trigo primero por el centro y después, con el avance de la conquista, hacia América del Sur (Ball 1930, 49-50) y la zona septentrional. ${ }^{13} \mathrm{~A}$ pesar de la existencia de numerosos problemas, la producción de trigo prosperó con un gran rendimiento en la meseta central mexicana debido a la calidad de las tierras y el clima tan favorable que había a una altitud comprendida entre los 1.500 y 2.000 metros (Del Río Moreno y López y Sebastián 1996, 36). ${ }^{14}$ Sin embargo, no ocurrió lo mismo en otras regiones donde los intentos de aclimatación del trigo fallaron una y otra vez debido, sobre todo, a las condiciones geoclimáticas imperantes. A pesar de ello, la búsqueda e introducción de variedades más adaptadas y resistentes con un gran potencial para su producción comercial, fue constante (de León Meza 2013). Todos estos esfuerzos fueron de gran importancia para el caso que ahora nos ocupa, ya que hicieron posible llevar hasta Nueva California trigos bien adaptados a una amplia gama de condiciones, sobre todo a climas secos y calurosos. En 177015 había ya en el continente norteamericano variedades adaptadas que proporcionaron una buena base para el trabajo que más adelante llevarían a cabo los mejoradores estadounidenses (Ball 1930, 50) ${ }^{16}$.

De todas las variedades que llegaron a Nueva California, la más importante variedad cultivada en los primeros años de colonización fue la Little Club, un trigo blanco de ascendencia española que posteriormente se llevó hasta el valle de Willamette y en 1859 a la zona de Walla Walla (Shepherd 1980, 53) 1718.

\section{Agricultura e Historia Natural}

Durante esta etapa inicial que hemos situado entre las primeras oleadas de inmigrantes a la costa noreste americana, hasta la guerra civil estadounidense, tanto en la costa este como oeste -recordemos que el centro no había sido prácticamente ocupado- la búsqueda, descripción, clasificación de y experimentación con las variedades que tenían a la mano, en busca de aquellas mejor adaptadas a cada contexto 
ecológico, geográfico y social, fue fundamental. Una búsqueda y experimentación posible gracias a la introducción de nuevas variedades por parte de los colonos en su desembarco en tierras americanas, al comercio internacional de semillas, a la adquisición y desarrollo de catálogos, al intercambio de información y a las expediciones científicas.

Ahora bien, allende de las variedades introducidas, también se generaron y utilizaron otras nuevas. Muchas de ellas surgieron de manera espontánea al mezclarse, de manera natural, distintas variedades producidas en zonas relativamente cercanas. Otras, fueron el resultado de selecciones artificiales amparadas en experiencias propias, locales o acumuladas durante generaciones en granjas o establos europeos. En cualquiera de los casos, el ojo entrenado del agricultor para percibir las diferencias y su actitud experimental, fueron fundamentales para el incremento en el número de variedades disponibles y, con ello, para el hallazgo de formas mejor adaptadas a cada contexto particular.

Nos encontramos, por consiguiente, en un contexto en el que el conocimiento de los granjeros era aún principalmente empírico, fruto de experiencias propias o cercanas, basadas en muchas ocasiones en el ensayo y error, en el que los distintos campos biológicos aportaban más descripciones y catálogos, que explicaciones propiamente dichas.

Mientras esto ocurría en los campos de labor, en el ámbito científico poco a poco se intentaba abandonar una ciencia dominada por la Historia Natural -propia del colonialismo tardío- en aras de nuevos modelos que permitieran no sólo describir, catalogar, introducir y experimentar con nuevas variedades (prácticas que nunca se abandonarán debido a su gran importancia práctica) - si no que también facilitara una mejor explicación de los fenómenos biológicos (y con ellos agrícolas), un mayor poder predictivo y, sobre todo, un mejor control de las distintas variables que estaban en juego.

Bajo esta nueva concepción de la ciencia, de corte claramente baconiano, la identificacón y producción de nuevas variedades siguió jugando un papel extraordinario. En el caso de la agricultura, la nueva ciencia se vió reforzada por un especial afán de control y predicción de cada aspecto del proceso productivo ${ }^{19}$. Este interés, evidente en los grandes hacendados coloniales en las décadas que rodearon la guerra de independencia ${ }^{20}$, estuvo también presente y fue uno de los principales elementos que hicieron posible la expansión de la producción triguera a espacios hasta entonces imposibles. Además, fue parte fundamental en el proceso de estandarización y normalización que dará como resultado la consolidación -no sin dificultades- de una producción agrícola comercial a gran escala.

\section{La expansión productiva y territorial del trigo estadounidense}

En el periodo de 1839-1909 la producción de trigo en los Estados Unidos se incrementó 7,54 veces (2.9\% por año), pasándose de 84 millones de fanegas ${ }^{21}$ aproximadamente al año, a 640 millones. En 1839 el centro de producción de trigo 
estaba localizado en el este de Wheeling (oeste de Virginia), Ohio y el norte del estado de Nueva Inglaterra y muy poco -relativamente hablando- en el oeste, como Illinois. Pero para 1909 el centro de producción se había movido alrededor de 800 millas hacia el oeste, a las zonas fronterizas de lowa y Nebraska. Las áreas centrales de la zona triguera moderna habían surgido -como podemos ver- en un área que se extiendía desde Oklahoma y Kansas en el sur, hasta Dakota del norte y las praderas canadienses en el norte por un lado; y una importante concentración en el interior del noroeste del Pacífico por otro. El desplazamiento occidental fue tan abrumador, que ninguna de las "nuevas áreas" había sido incluida en el famoso mapa de Parker y Klein de 1839. Sin embargo, para 1909 aportaban el 64\% de la producción. Al área occidental de los Apalaches, que producía menos del $50 \%$ de la producción triguera en 1839, proveyó el 92\% de la producción de 1909 (Olmstead y Rhode 2008, 23-24).

\section{¿Por qué y cómo fue posible este desplazamiento?}

Antes de ver cómo fue la selección e introducción de variedades en los dos principales movimientos de expansión territorial y productiva del trigo, es pertinente preguntarse cómo y porqué se dio dicha expansión. Una cosa es que la identificación, creación e introducción de variedades ayudara, hiciera posible la expansión territorial $y$, otra muy distinta, que fuera causa de la misma.

En ese sentido, si la primera mitad del siglo XVIII se había caracterizado por un crecimiento y desarrollo constante, tanto económico como poblacional, la segunda mitad lo hizo por la multiplicación de extensos asentamientos franceses en la zona de Illinois y los estados adyacentes (Ball 1930, 52) y los grandes contingentes de irlandeses, alemanes y escandinavos que fueron al Nuevo Mundo en busca de oportunidades y un nuevo espacio vital (Doval 2009, 12).

A inicios del siglo XIX el proceso de industrialización en Inglaterra primero, y de otros países europeos después, hizo que el modo de producción agrícola de muchas regiones europeas resultara insostenible ante el avance de la agricultura intensiva. Una agricultura que dejaba como única alternativa a quienes tan sólo conocían modelos previos, la migración. Así, grandes masas de agricultores europeos migraron nuevamente al continente americano con el sueño de un trabajo y, en muchos casos, de nuevas tierras (Portes \& Rumbaut, 2014; Doval 2009, 12).

Ahora bien, la llegada de estas masas de trabajadores, principal pero no exlusivamente europeos, cambiaron muchas de las condiciones laborales y sociales de quienes allí ya vivían. Ante estas, y convencidos de la democracia igualitaria, masas de trabajadores nacidos en el continente americano se sumaron a unas caravanas que fueron moviendo la frontera a gran velocidad (Doval, 2009). Unos y otros tenían como principal objetivo ir hacia el oeste y tomar ventaja en la posesión de nuevas tierras a la luz de Ley de Asentamientos Rurales ${ }^{22}$.

Les impulsaba además en la mayoría de los casos una creencia compartida: en el esfuerzo y el trabajo personal estaba la recompensa, el medio para lograr la prosperidad. Todos tenían que trabajar para ganarse la vida y, bajo esta convicción, 
muchos de quienes marcharon hacia el oeste, lo hicieron como rechazo al gobierno de los gentileshombres que, con mayor velocidad y fuerza, estaban ocupando puestos de poder. Entre ello, especial rechazo generaban a aquellos que no trabajaban directamente las tierras con las que se estaban enriqueciendo.

Los agricultores querían ser libres, tener sus propias tierras y no tener que responder a los caprichos de hacendados, científicos o funcionarios ${ }^{23}$. Ahora bien, ¿a qué tipo de caprichos se referían? La respuesta está relacionada con otro de los motivos que empujó a muchos agricultores, e incluso dueños de tierras, a dejar atrás éstas en busca de otras más orientales: el agotamiento de los suelos.

En ese sentido, fueron numerosas las voces que lamentaban la falta de progreso de la agricultura estadounidense (comparada sobre todo con la europea) y los numerosos riesgos que ello comportaba. George Logan denunció en 1818 en ese sentido ante la Philadelphia Society for Promoting Agricultura (PSPA) ${ }^{24}$, cómo los campesinos, en lugar de buscar nuevos y mejores métodos de cultivo, seguían prácticas tradicionales que habían aprendido de sus padres (Minella 2016), unas prácticas que no sólo les impedían lograr el mayor cultivo posible, sino que también iban destruyendo la fertilidad del suelo. De ahí que -señalaba- con el paso de los años y el desgaste de las tierras, numerosos agricultores se vieran en la necesidad de abandonar éstas y buscar campos frescos en Occidente, drenando de ese modo los recursos de los estados del este (Minella 2016, 435).

Frente a esta situación, miembros de las recientemente constituidas sociedades agrícolas adviertieron que los agricultores debían practicar una agricultura de permanencia que permitiera a las futuras generaciones ganarse la vida en el mismo terreno. Estos caballeros -como seguían viéndoles los agricultores- abogaron por la educación agrícola como un medio para hacer de la agricultura una profesión honorable. Ello señalaba, entre otras cosas, la necesidad de generar nuevos modelos de producción agrícola basados en la ciencia.

Nos encontramos, por tanto, en mitad de un periodo de gran expansión territorial con al menos dos posiciones claramente diferenciadas, pero no siempre opuestas. Por un lado, quienes seguían con sus prácticas tradicionales -en muchas ocasiones importadas por los migrantes recién llegados de Europa- y, por otro, con quienes apostaban por el desarrollo e improvement. La búsqueda de modelos y estrategias de comunicación entre unos y otros fue, a la postre, clave en el posterior desarrollo agrícola, económico, político y social del país (Minella 2016, 435-436). ${ }^{25}$

Retornando a la cuestión de la expansion geográfica de la producción triguera, una de las cosas que llaman inmediatamente la atención son las grandes diferencias existentes entre las distintas regiones en las que se produjo esta ocupación territorial. Diferencias tan grandes desde el punto de vista geoclimático como las que se observa entre continentes distintos (Olmstead y Rhode 2008, 23-24). De ahí que -señalaba Carleton (1900)-, el desplazamiento de la producción de trigo no fuera sólo el resultado de una simple importación o transferencia de conocimientos o técnicas productivas, sino que se debió principalmente a la adecuada selección y mejoramiento de variedades de trigo. Con esta selección se buscaba, ante todo, mejorar el rendimiento por 
unidad de superficie; la calidad y tamaño del grano; su accesibilidad, textura y valor alimenticio; la adaptación de la planta a su entorno físico y fisiológico; y la protección de la cosecha ante enfermedades y plagas (Ball 1930, 48-49).

Olmstead y Rhode precisan en ese sentido que la producción de trigo en las nuevas regiones se debió sobre todo a la introducción de nuevas variedades de trigo duro rojo completamente diferentes a las variedades producidas en las viejas áreas. De hecho, en la mayoría de las ocasiones, ni siquiera se conocían estas variedades en EEUU antes de 1839 (Olmstead y Rhode 2008, 24).

Veamos con un poco de atención cómo los dos principales movimientos de la expansión terriorial de la producción triguera, una hacia las grandes praderas del norte y otra hacia el oeste, estubieron siempre acompañados y fueron posibles, gracias en buena medida, a la selección e introducción de variedades.

\section{Hacia las praderas del Norte}

La conquista de las praderas del norte no fue, al menos desde el punto de vista agrícola, sencillo. Aquellos pioneros que intentaron introducir el cultivo del trigo en las nuevas regiones, sufrieron repetidas pérdidas en sus cosechas, ya que las variedades cultivadas comunmente en la región oriental, no se adaptaban a las condiciones normales de las Grandes Llanuras ${ }^{26}$. La identificación, prueba y selección de las variedades mejor adaptadas en cada contexto particular fue esencial. Así, mientras en el este del Mississippi había prevalecido históricamente la producción de trigo blando -tanto blanco como rojo-, fue el trigo duro rojo el que terminaría dominando en las Grandes Praderas ${ }^{27}$. Este trigo duro, que devino popular en las regiones norteñas de las Grandes Praderas después de 1900, era una especie diferente del trigo común, con una calidad y propiedades de harina diferentes y por tanto de usos (Olmstead y Rhode 2008, 26-27).

Siguiendo el extraordinario trabajo de K. S. Quisenberry and L. P. Reitz podemos reconstruir la colonización triguera de los campos americanos y ver que el trigo se cultivó por primera vez en Kansas en la década de 1830 y principios de 1840; los indios de Delaware ya lo cultivaban en 1835 a la orilla norte del Kaw; en 1840 se cultivaba en el condado de Johnson, en 1843 en la Shawnee Friends Mission, en 1844 en el condado de Doniphan y en Texas en torno a 1833. Parece ser que llegó a Nebraska en 1853 o 1854, si bien la agricultura no se desarrolló en esta zona hasta la década de 1870 (Quisenberry y Reitz 1974, 99). Esta difusión exitosa del cultivo del trigo a través de los vastos territorios, extendiéndose desde el Panhandle de Texas hasta las praderas canadienses, fue posible, como ya hemos señalado, gracias a la introducción del trigo rojo de invierno y el trigo duro de primavera, algo totalmente nuevo en América del Norte (Olmstead y Rhode 2008, 26-27). En 1842 David y Jane Fife de Otonabee (Ontario) cultivaron Red Fife (variedad idéntica a la conocida como Gallega en Europa), la primera variedad de trigo duro de primavera cultivada en América del Norte (Olmstead y Rhode 2008, 26-27). Muy pronto esta variedad de primavera se convirtió en la base para la expansión de la frontera trigera en Wisconsin, Minnesota, 
Dakota y Canadá. También fue la matriz desde la que se desarrollaron posteriormente nuevas variedades de trigo, como el Marquis. En las primeras encuestas fiables sobre las variedades de trigo cultivas en 1919, Dakota del Norte, Dakota del Sur y Minnesota, se percibe cómo el trigo duro de primavera y el trigo duro rojo, excluyeron virtualmente todas las demás variedades (OImstead y Rhode 2008, 26-27).

Los primeros trigos cultivados en Nebraska fueron las variedades de primavera Java, Red Fife y Bluestem, mismas variedades que se cultivaron también en el este de Kansas junto a variedades de invierno suaves de color rojo como el Red May (primera variedad cultivada en Texas), Mediterranean, Lancaster, Fultz y Zimmerman (Quisenberry y Reitz 1974, 100).

El otro gran protagonista fue el Turkey Wheat introducido por los Menonitas, quienes tubieron éxito allí donde otros habían fracasado. La razón de ello la encontramos en el hecho de que de los primeros asentamientos de Menonitas en Kansas en 1873 fueron precedidos por la visita al continente americano de observadores que se habían adelantado buscando una tierra y un clima similar al de sus tierras rusas. Dichas tierras las encontraron en Kansas y Nebraska. Además, cuando los Menonitas llegaron tenían ya experiencia en el cultivo en grandes praderas, aparecieron con algo de equipo adecuado, know-how y una variedad de trigo -el Turkey Wheat- que se adaptaba muy bien a ese medio ambiente (Quisenberry y Reitz 1974, 103-104).

La aceptación del Turkey Wheat fuera de la comunidad de Menonitas fue lenta debido a la apariencia del grano y al hecho de que los molinos estadounidenses no estubieran preparados para un trigo tan duro (Quisenberry y Reitz 1974, 105). Sin embargo, al menos dos razones llevaron a los molineros a hacer los ajustes que fueran oportunos para poder molerlo; era mucho más barato que el trigo blando y tenía un alto contenido de gluten, algo que hacía que su demanda fuera muy alta (Quisenberry y Reitz 1974, 106).

Poco a poco el Turkey Wheat se convirtió en una de las principales variedades de trigo cultivadas en Estados Unidos y base sobre la cual se llevarían a cabo muchos de los trabajos de desarrollo de nuevas variedades. Ahora bien, esta introducción y expansión del Turkey Wheat, como la de todas las demás variedades introducidas desde 1602 en Buzzards Bay, fueron posibles gracias a enormes esfuerzos y prácticas basadas en la experiencia y la tradición. No encontramos todavía, por tanto, una investigación científica de campo o la aplicación de nuevas práctivas o conocimientos provenientes de una ciencia moderna.

\section{Introducción de variedades en los territorios mexicanos anexados}

En 1882, treinta y cuatro años después de la firma del Tratado de Guadalupe Hidalgo y fin de la Guerra mexicana-estadounidense, se introdujo en los antiguos territorios mexicanos el Pacific Bluestem, un trigo australiano común de primavera ${ }^{28}$. Esta variedad tuvo tal éxito que en 1890 era ya el trigo líder en aquellas zonas con precipitaciones bajas. De las distintas variedades que agricultores, compradores de grano y empresas molineras fueron introduciendo, los principales granos que 
compitieron con el Little Club -recordemos que durante la epoca colonial había sido la más importante- en las zonas de precipitaciones superiores, fueron dos trigos de invierno, el Fortyfold (también llamada moneda de oro) llevado desde el valle de Genesee en Nueva York y el Russian Red, un trigo de origen inglés que rendía bien pero cuyo grano era de muy baja calidad. El Chaff Red, un trigo de primavera similar al Little Club, fue introducido alrededor de 1900 en aquellas zonas con precipitaciones medias (15 a 20 pulgadas por año) así como el Jones Fife, un híbrido de invierno de Nueva York, en la región de Walla Walla. El Pacific Bluestem siguió cultivándose en aquellas zonas de precipitaciones inferiores donde el Turkey Red fue, tras su expansión desde los Grandes Valles, su principal rival. Muchas otras variedades fueron llegando a esta zona con menos éxito que las anteriores, si bien vale la pena destacar dos trigos de primavera: el Jenkins y el Early Wilbur (Shepherd 1980, 54).

Si bien el Little Club tenía una baja resistencia al invierno, de ahí los intentos de introducción de otras variedades, a medida que se fueron evaluando estas últimas se vio la necesidad de conservarlo y seguir cultivándolo. De hecho, tanto el Little Club como el Pacific Bluestem fueron las variedades que dominaron la región al menos hasta 1890. A partir de esta fecha, el Little Club tuvo principalmente dos usos: como resorte, como trigo de otoño y como base sobre la que se desarrollaron nuevos híbridos. ${ }^{29}$

Los trigos cultivados en los climas más cálidos, como California u Oregón, se sembraban en primavera y se cosechaban en otoño. Los productores del este de Washington, con un verano más corto, sembraban en otoño para conseguir hasta un $21 \%$ más que las plantaciones de primavera al contar con un tiempo de crecimiento mayor (Carlson 2005, 56).

Como podemos ver, la principal lógica de actuación en estos territorios fue la misma que encontramos en la costa este y las grandes llanuras centrales: la identificación de las variedades mejor adaptadas a cada entorno junto al desarrollo de buenas prácticas culturales ${ }^{30}$ y el control de plagas y enfermedades. La falta de una teoría que permitiera explicar, comprender y orientar la práctica, no era sólo casi inexistente, sino considerada a veces como irrelevante. Ello es algo que permanerá en parte hasta la última década del siglo XIX y las primeras del siglo XX, momento en el que se dieron los primeros exitos en la introducción de una base científica (matemática y teórica) en el desarrollo de nuevas variedades. Veamos esta relación entre teoría y práctica con un poco más de detalle.

\section{Teoría y práctica}

Como hemos podido ir viendo, el éxito de la producción de trigo dependió entre los siglos XVIII y XIX de muchos factores, entre los que -siguiendo a Carlson (2005, 55)- podemos destacar ahora mismo tres: contar con grandes extensiones de tierra, disfrutar de un clima favorable y tener acceso a mercados. En las nuevas tierras de producción triguera -sur y sobre todo oeste americano- los agricultores tenían por lo general grandes extensiones de tierra y acceso a un importante número de líneas de ferrocarril. ${ }^{31}$ Ahora bien, a pesar de las facilidades que estas daban, las explotaciones 
seguían encontrandose -comercialmente hablando- distantes de los mercados europeos y debían competir con el aumento de áreas cultivadas en Argentina, India y Pakistán (Carlson 2005, 56; Serrano-Bosquet y Rivas-Sada 2018, 235).

Si el aumento de la competitividad internacional y la presión que ello generaba, así como las dificultades que las diferencias geoclimáticas introducían, hicieron a los empresarios, banqueros y agricultores volver sus miradas hacia la ciencia, hacia las estaciones experimentales con el fin de intentar ser más competitivos (Carlson 2005, 56), hasta finales del siglo XIX la introducción, selección y producción de variedades de trigo siguió siendo fruto, en gran medida, de una práctica basada principalmente en la intuición y la experiencia. Ello no significa, tal y como hemos ido indicando, que no hubiera interés científico, ni tampco experimentación. Como muy bien señala True en su famoso trabajo de 1937, hubo y se fomentó la investigación agrícola desde muy tempranas fechas. Al menos, desde finales del siglo XVIII, donde encontramos personajes como Washington o Jefferson diseñando y llevando a cabo experimentos a partir de la lectura de libros recientemente publicados en Europa; o los grandes esfuerzos individuales y colectivos alentados, promocionados y publicitados por las Sociedades Agrícolas.

Los productores e investigadores tenían a su disposición númerosos datos e información procedentes de su experiencia y experimentos ${ }^{32}$. Pero, hasta finales del siglo XIX, y sobre todo inicios del XX con la recuperación de las leyes de Mendel, no tenían una forma oportuna, moderna, de interpretar dichos datos de manera concluyente. Y, muchos menos, de controlar los procesos. Ni siquiera se va a conseguir inicialmente en los numerosos centros de investigación agrícola creados por los estados y la Federación entre 1840 y $1888 .^{33}$ Las cosas empezarían a cambiar gracias a la aprobación de la Hatch Experiment Station Act en 1887 (Carlson 2005, 56), las nuevas noticias e información científica que llegaban de Europa y, sobre todo, a trabajos como los llevados a cabo por investigadores como W.J. Spillman, un joven profesor de agronomía en el Washington State Agricultural College and School of Science (Palouse) que, por primera vez en el mundo, corroboró independientemente las tesis de Mendel (Spillman 1902a). ${ }^{34}$ Incluso, antes del redescubrimiento de las mismas por los europeos en 1900, las aplicó -ignorando su valor- en programas de investigación y en exposiciones dirigidas a un público agrícola (Carlson 2005, 69)..$^{35}$ Su más importante contribución fue observar y demostrar que era posible predecir qué tipos podrían resultar del cruce de dos variedades establecidas, así como la proporción aproximada de cada tipo que lo haría en la segunda generación (Spillman 1902a). Se abría así la puerta a la posibilidad de crear nuevas formas de plantas y animales de manera más controlada. Ahora bien, tal y como el mismo Spillman llegó a denunciar, el principal problema del campo estadounidense no era la falta de experimentación, si no de interés por la teoría: lo único que interesaba en la selección y cruce de variedades parentales, era replicar características útiles (Carlson 2005, 68-69). 


\section{Reflexiones finales}

Como hemos estado viendo, los intentos de estandarización y normalización de la producción de trigo durante el periodo revisado, la introducción de nuevas variedades y los esfuerzos para mejorar las existentes, fueron en su gran mayoría el resultado de conocimientos empíricos y selecciones artificiales basadas en experiencias personales y comunitarias, sin un modelo matemático y estadístico que permitiera la predicción y el control del proceso de cruzamiento. Sin embargo, ello no significa que se trataran de meros cruzamientos intuitivos sin una base lógica. Junto a la experiencia personal y comunitaria, ya de por sí con una lógica propia que deberemos desarrollar en otros trabajos, encontramos también esfuerzos ligados con la ciencia que entonces se tenía a mano. Una ciencia o ciencias de la vida ligadas a la Historia Natural y los nuevos descubrimientos y conocimientos - procedentes principalmente de Europa- que desde otros campos iban llegando.

Los ensayos, llevados a cabo en algunas ocasiones por agricultores individuales, en otras, de manera comunitaria y, en muchas otras, por o desde las sociedades agrícolas e instancias gubernamentales, generaron una gran cantidad de conocimientos con los que fue posible el desarrollo de la agricultura comercial a gran escala del siglo XX. Ahora bien, no fueron sólo los pequeños, medianos y grandes productores privados los que llevaron a cabo estos esfuerzos. Desde las mismas estancias gubernamentales se intentó obtener variedades de trigo de muy distintas partes del mundo (Cary 1857, 1), distribuirlas y educar a los productores para que las utilizaran (Willard, 1857; Cary F. G., 1857; Webster, 1857). En su ya clásico trabajo, Ball constata cómo, ante la evidencia de que una de las formas más rápidas y seguras de mejorar el trigo americano parecía residir en la introducción de nuevas variedades de países extranjeros, especialmente cuando se estaba estableciendo un territorio tan nuevo y diferente, los oficiales consulares y navales estadounidenses en el extranjero, siguiendo el ejemplo de Franklin antes de la Revolución, habían estado enviando material agrícola de vez en cuando. El resultado de ello fue la introducción de un gran número de variedades de trigo de muchos países diferentes del mundo que, junto a una creciente inmigración de Europa Central, incorporó muchas otras variedades de trigo (Ball 1930, 54).

Así, fruto las distintas prácticas, experiencias y lógicas, fue posible la creación de un extraordinario banco -no siempre oficial- de variedades de trigo con las que el agricultor, en principio, podía contar. El número y una calidad de variedades adaptadas a tan dispares condiciones geoclimáticas, facilitaron enormemente la gran expansion territorial y la constitución de una gran agricultura comercial que gozó, al menos hasta la gran crisis de la última década del siglo XIX, de una gran posición internacional. Detrás de esta nueva agricultura comercial encontramos, por tanto, una reorientación agrícola posibilitada y condicionada por la experiencia e innovación biológica previa y subordinada, en buena medida -al menos durante las útimas décadas-, por la gran competencia internacional que dichos modelos habían generado.

El cambio de enfoque -de un modelo agrícola de subsistencia o autoconsumo, a otro con miras al comercio internacional- supuso, entre otras cosas, un cambio (y complejización) en los criterios de toma de decisión. Si, durante los primeros 
intentos de introducción y luego expansión territorial de la producción de trigo, los principales criterios de selección de variedades de semillas fueron geobiológicos (resistencia a las plagas, clima, adaptabilidad), los criterios de adaptación a las técnicas y necesidades de producción industrial (desde el diseño del molino hasta las innovaciones en conservación y transporte), las condiciones de uso y las demandas de los diversos mercados locales e internacionales, empezaron a ganar importancia. Este cambio coincidió con el proceso de industrialización y consolidación del pragmatismo norteamericano que, habiendose iniciado en las colonias de Nueva Inglaterra, permeó y determinó el modelo de producción agrícola que dominó la mayor parte del siglo XX.

Junto a esta actitud pragmática, una de las características más importantes que hemos observado a lo largo de este proceso de desarrollo de la agricultura científica norteamericana, la introducción, el mejoramiento y la producción de variedades no solo fue una práctica común, si no una parte fundamental, también, del propio modelo. La misma dinámica y preferencia la encontramos presente en las primeras introducciones (condicionadas por los antecedentes de los inmigrantes), luego en los procesos más complejos de búsqueda, identificación y clasificación durante el periodo de expansión territorial (propios de la historia natural) ${ }^{36} \mathrm{y}$, finalmente, en el periodo de mejora y producción de nuevas variedades (híbridos) a través de la selección artificial.

En el siglo $X X$, al igual que en los tres siglos anteriores, la introducción y creación de nuevas variedades siguió transformando el paisaje agrícola norteamericano. Unas prácticas que fueron cruciales, tal como lo señala Samuel Hays (1957), para la agricultura en general y -nos atrevemos a agregar- la producción de trigo en particular. Ésta -tal y como hemos desarrollado y demostrado en trabajos previos (Serrano-Bosquet y Rivas-Sada 2018, 254-255)-, pasó de ser una operación relativamente simple, a una actividad altamente compleja que exigía inversión en investigación, equipamiento, información científica y conocimiento del mercado. La agricultura se fue enredando cada vez más con el complejo sistema industrial (Hays 1957, 18) y, en consecuencia, el trabajo arduo que había definido a los primeros pobladores, pronto resultó insuficiente. Los agricultores tuvieron que desarrollar una mentalidad más orientada hacia los negocios, calculadora, consciente y siempre informada del estado de los mercados internacionales (Serrano-Bosquet y Rivas-Sada 2018, 254-255).

Esta perspectiva, que se desarrolló durante el período que abarca este trabajo sería, junto con una creciente dependencia de la ciencia, la especialización, la estandarización y la normalización de todos los aspectos de la producción, la principal característica del modelo agrícola norteamericano que se exportó y dominó en gran medida la agricultura de todo el mundo durante la segunda mitad del siglo XX. Un modelo que, como trataremos de demostrar en trabajos posteriores, se mantiene vigente hasta nuestros días. 


\section{Referências}

BALL, Carleton R. 1930. "The History of American Wheat Improvement." Agricultural History 48-71. Accessed 05 27, 2015. http://www.jstor.org/stable/3739359.

BAYLES, B.B., and J. Allen Clark. 1954. "Classification of Wheat Varieties Grown in the United States in 1949." Edited by UNITED STATES DEPARTMENT OF AGRICULTURE. Technical Bulletin (No. 1083): 1-180. Accessed 05 28, 2015. http://naldc.nal.usda.gov/ naldc/download.xhtml?id=CAT86201072\&content=PDF.

BIDWELL, Percy W. 1921. "The Agricultural Revolution in New England." The American Historical Review (Oxford University Press on behalf of the American Historical Association) Vol. 26 ( $\mathrm{N}^{\circ}$ 4): 683-702. Accessed 05 23, 2015. http://www.jstor.org/ stable/1836733.

CÁRCER Y DÍSDIER, Mariano. 1953. Apuntes para la historia de la transculturación indoespañola. México: Instituto de Historia.

CARLETON, M.A. 1900. The basis for the improvement of American wheats. Div. Veg. Phys. Path. Bull, USDA, Washington, DC: U.S. Gov. Print.

CARLSON, Laurie. 2005. "Forging His Own Path: William Jasper Spillman and Progressive Era Breeding and Genetics." Agricultural History (Agricultural History Society) Vol. 79 $\left(n^{\circ}\right.$ 1): 50-73. Accessed 05 25, 2025. http:/www.jstor.org/stable/3744877.

CARY, F. G. (ed.). 1857. "The Agricultural Department of Our Government - Distribution of Seeds, Etc." The Cincinnatus (Farmer's College) Vol. 2, n 1 (january, 1): 1-6. https:// archive.org/stream/cincinnatus00carygoog\#page/n12/mode/2up.

CARY, F. G. 1857. "Science and the Farmer." The Cincinnatus Vol. 2 (1): 7-9. Obtenido de https://archive.org/stream/cincinnatus00carygoog\#page/n12/mode/2up.

COTTER, Joseph. 1994. "The Origins of the Green Revolution in Mexico. Continuity or Change?" In Latin America in the 1940s. War and Postwar Transitions, by David Rock, 224-247. Berkeley: University of California Press.

2003. Troubled Harvest: Agronomy and Revolution in Mexico, 1880-2002. Westport, Connecticut: Praeger.

CUTTER, Donald C. 1987. "Pedro Alberni y los primeros experimentos de agricultura científica en la costa Noroeste del Pacífico." Revista de Historia Naval (Instituto de Historia y Cultura Naval - Armada Española) Año V (18): 41-55.

DE LEÓN MEZA, C. René. 2013. "La agricultura del trigo y su mano de obra en Nueva Galicia, siglo XVII." XIV Congreso Internacional de Historia Agraria.

BRENNER, Robert. 2003. Merchants and revolution: Commercial change, political conflict, and London's overseas traders ; 1550 - 1653. Princeton, New Jersey: Princeton Univresity Press. 
BRENNER, Robert (2007) "Property and progress: Where Adam Smith went wrong," in Chris Wickham (ed.) Marxist History-Writing for the Twenty-First Century. Oxford: Oxford University Press: 49-111.

DEL RÍO MORENO, Justo L., and Lorenzo E. López y Sebastián. 1996. "El trigo en la ciudad de México. Industria y comercio de un cultivo importado <1521-1564)." Revista Complutense de Historia de América Vol. 22: 33-51. Accessed 05 28, 2015. http:// revistas.ucm.es/index.php/RCHA/article/viewFile/RCHA9696110033A/28995.

DESTLER, Chester McArthur. 1968. "Forward Wheat'for New England: The Correspondence of John Taylor of Caroline with Jeremiah Wadsworth, in 1795." Agricultural History 42 (3): 201-210. Accessed 03 29, 2017. http://www.jstor.org/stable/37.

DOVAL, Gregorio. 2009. Breve historia de la conquista del Oeste. Madrid: Nowtilus.

FAIRCHILD, David G. 1905. "Plant and Animal Introduction." American Breeder's Association, Proceeding. Ithaca, New York: Cornell University, Mann Library. 92-100.

FITZGERALD, Deborah. 1986. "Exporting American Agriculture: The Rockefeller Foundation in Mexico, 1943-53." Edited by Ltd. Sage Publications. Social Studies of Science 16 (3): 457-83.

HAYS, Samuel P. 1957. The Response to Industrialism: 1885-1914 . Chicago: University of Chicago Press.

LNSTITUTO JALISCIENSE DE ANTROPOLOGÍA E HISTORIA. 1970. Actas de Cabildos de la ciudad de Guadalajara. Guadalajara: Ayuntamiento Constitucional de Guadalajara, Vol. Primero, 1607- 1635.

MINELLA, Timothy K. 2016. "A Pattern for Improvement: Pattern Farms and Scientific Authority in Early Nineteenth-Century America." Agricultural History 90 (4): 434-458. doi:10.3098/ah.2016.090.4.414.

OLMSTEAD, Alan L., and Paul W. Rhode. 2008. Creating Abundance. Biological Innovation and American Agricultural Development. Cambridge: Cambridge University Press.

PORTES, Alejandro, and Rubén G. Rumbaut. 2014. Immigrant America : A Portrait. Fourth edition, revised, updated, and expanded. Oakland, Berkeley and Los Angeles: University of California Press. Accessed 06 03, 2019.

POST, Charles. 2009. "Agrarian Class Structure and Economic Development in Colonial British North America: The Place of the American Revolution in the Origins of US Capitalism." Journal of Agrarian Change, 9(4), 453-483. https://doi.org/10.1111/j.14710366.2009.00228.x

QUISENBERRY, K. S. , and L. P. Reitz. 1974. "Turkey Wheat: The Cornerstone of an Empire." Agricultural History Vol. 48 (No. 1, Farming in the Midwest, 1840-1900: A Symposium): 98-110. Accessed 03 19, 2015. http://www.jstor.org/stable/3741420. 
SCHWARTZ, Amy D. 1995. "Colonial New England Agriculture: Old Visions, New Directions." Agricultural History (Agricultural History Society) Vol. 69 ( $n^{\circ}$ 3): 454-481. Accessed 06 09, 2015. http://www.jstor.org/stable/3744338.

SERRANO-BOSQUET, Francisco Javier, and Eva Rivas-Sada. 2014. "El lugar de la Filosofía de la agronomía dentro del campo de la Filosofía de las ciencias de la vida." Metatheoria - Revista de Filosofía e Historia de la Ciencia Vol. 5 (1): 175-186. http:// www.metatheoria.com.ar/index.php/m/article/view/140 .

SERRANO-BOSQUET, Francisco Javier, and Eva Rivas-Sada. 2018. "William J. Spillman. Teoría y práctica en la producción de trigo estadounidense." Historia 396 Vol. 8 (1): 225-260. http://www.historia396.cl/index.php/historia396/article/view/246/122.

SHARP, Paul, and Weisdorf, Jacon. 2013. "Globalization revisited: Market integration and the wheat trade between North America and Britain from the eighteenth century." Explorations in Economic History, 50(1), 88-98. https://doi.org/10.1016/j.eeh.2012.08.002.

SHEPHERD, James F. 1980. "The Development of New Wheat Varieties in the Pacific Northwest." Agricultural History Vol. 54 (n 1, Agricultural History Symposium: Science and Technology in Agriculture): 52-63. Accessed 05 28, 2015. http://www.jstor.org/ stable/3742593.

SPILLMAN, William Jasper. 1902a. "Quantitative Studies on the Transmission of Parental Characters to Hybrid Offspring." Edited by A. C. True, W. H. Beal and H. H. Goodell. Fifteenth Annual Convention of the Association of American Agricultural Colleges and Experiment Stations. Washington, D.C.: USDA. 88-98. https://archive.org/details/ proceedingsoffif115asso.

SUÁREZ, Clara Elena. 1985. La política cerealera y la economía novohispana: el caso del trigo. México: CIESAS.

TAYLOR, Paul S., and Anne Loftis. 1981. "The Legacy of the Nineteenth-Century New England Farmer." The New England Quarterly (The New England Quarterly, Inc.) Vol. 54 ( $n^{\circ} 2$ ): 243-254. Accessed 05 23, 2015. http://www.jstor.org/stable/364972.

TRUE, Alfred Charles. 1937. History of Agricultural Experimentation and Research in the United States, 1607-1925. Washington, DC: USDA, Misc. Publn 251.

WEBSTER, Daniel. 1857. "Education of the Agriculturist." The Cincinnatus Vol. II (2): 58. https://archive.org/stream/cincinnatus00carygoog\#page/n12/mode/2up.

WESSEL, Thomas R. 1976. "Agriculture, Indians, and American History." Agricultural History Vol. 50 (n¹): 9-20. http://www.jstor.org/stable/3741903.

WILLARD, O.A. 1857. "Education of Farmers." The Cincinnatus Vol. 2 (5): 221-223. https:// archive.org/stream/cincinnatus00carygoog\#page/n12/mode/2up.

ZELINSKY, Wilbur. 1958. "The New England Connecting Barn." Geographical Review Vol. 48 ( $\left.n^{\circ} 4\right)$ : 540-553. http://www.jstor.org/stable/211674. 


\section{Notas}

1 Pueden encontrar más información sobre la aparición de este enfoque y la visión económica previa basada en el desarrollo mecánico en el trabajo de Alan L. Olmstead y Paul W. Rhode (2008).

2 Quienes estubieran interesados en profundizar en esta área, encontrará en el trabajo de Amy D. Schwartz (1995) una excelente revisión historiográfica sobre la agricultura colonial en Nueva Inglaterra. Aquí solo proporcionaremos algunas notas para contextualizar los esfuerzos de identificación y selección de las variedades de trigo realizadas durante este período.

${ }_{3}^{3}$ Probablemente el mejor ejemplo de ello sea la figura de John Rolfe quien nada más tocar suelo americano puso en marcha la producción de tabaco con fines comerciales con la metrópoli.

${ }^{4}$ Las consecuencias y los cambios que tuvieron que enfrentar fueron más allá de los relacionados con una simple transformación de los modelos de producción. Algunos de ellos se pueden encontrar en Paul S. Taylor y Anne Loftis $(1981,243)$.

5 Unos valores que derivaban directamente de la elección fundamental que hicieron los colonos recien llegados al nuevo continente de rechazo al patrón feudal europeo y de abrazo a un modelo de sociedad levantado sobre la imagen del agricultor independiente (Taylor y Loftis 1981, 243). El agricultor estadounidense de finales del siglo XVIII e inicios XIX creía aún en la igualdad de oportunidades y tenía un fuerte sentido de comunidad. Las Reuniones Agrícolas lo fomentaron mucho, así como más adelante, el intercambio de experiencias en revistas (Taylor y Loftis 1981, 243).

${ }^{6}$ Ello chocaba con la ética puritana tradicional que exigía a todos los miembros de la familia ser productivos y no sólo meros consumidores.

7 Esta situación puede entenderse un poco mejor si la situamos en su contexto: el nacimiento del capitalismo, y según numerosos historiadores, la "primera era de la globalización (Sharp \& Weisdorf 2013, 88-89), un periodo en el que, además (o precisamente por ello) termina por completarse el proceso de integración transatlántica del mercado de cereales (Post 2009, 453454). Si bien no es posible detenernos ni desarrollar este punto convenientemente, consideremos mínimamente los principales modelos explicativos emanados de las distintas posturas que los historiadores económicos y sociales han manifestado sobre el fenómeno, en las que atribuyen mayor o menor relevancia a la transición de una economía colonial a una economía local [de Estado/nacional] en el rápido crecimiento de la producción agrícola e industrial de los Estados Unidos en el siglo XIX. La primera postura, defendida principalmente a mediados del siglo XX por autores como Richard L. Bushman, Lewis C.

8 GRAY, Charles S. Grant, Raymond C. Loehr, JamesT. Lemon y Max G. Schumacher, aboga por una continuidad del desarrollo económico y social antes y después de la independencia (Post, 2009: 454). Casi todos estos autores coincidían en señalar que tanto la agricultura familiar de las colonias del norte, como las plantaciones esclavistas de las colonias del sur, eran formas de producción capitalista en la medida que supieron responder a las oportunidades de mercado y la producción especializada, acumularon tierras e introdujeron nuevas herramientas y métodos (Post, 2009: 454). Por otro lado, autores como Percy Bidwell, Christopher Clark, Alan Kulikoff, Michael Merrill y Eugene Genovese, han constatado cómo, durante el siglo XVIII, tanto los pequeños agricultores del norte, como la mayoría de los agricultores del sur que no poseían esclavos, eran esencialmente productores de subsistencia y que, en el mejor de los casos, vendían sus excedentes con el fin de poder comprar algunos bienes que ni ellos ni sus vecinos podían producir (Post, 2009: 454), lo que contradice la postura anterior. A estos dos grandes 
modelos, a los que vendrían a sumarse intentos de síntesis y pequeñas variaciones, merece la pena sumar la propuesta del mismo Charles Post (2009), y una versión más actualizada de Paul Sharp y Jacob Weisdorf (2013). Post, partiendo de Robert P. Brenner (1993 y 2007) enfatiza la necesidad de atender a las relaciones específicas entre productores y no productores durante el siglo XVIII (relaciones de clase), así como entre éstos y la propiedad de las herramientas y la tierra (proceso laboral). $Y$ es que - señalan- es a partir de estas relaciones que se fueron creando las "reglas de reproducción" que dieron forma a los modos en que los productores individuales respondieron a las fluctuaciones del mercado y la población (Brenner 2007) ya que estas fluctuaciones fueron las que, finalmente, marcaron el paso de transición de los modelos de producción colonial, al modelo capitalista decimonónico (Post 2009). Por su parte, Sharp y Weisdorf (2013), distinguen - utilizando el análisis de varianza y el modelo VAR - tres etapas en la constitución de la "primera era de la globalización", que supone la integración de los mercados transatlánticos de trigo: una primera etapa, que abarca todo el siglo XVIII, durante la cual el comercio fue relativamente libre (Sharp, 2010); la segunda, que se extiende desde 1800 hasta 1847, etapa durante la cual la Guerra Napoleónica y el alto nivel de protección que vivieron los mercados británicos condicionaron en gran medida el comercio internacional; la tercera de ellas se extiende desde la eliminación gradual de las Leyes del Maíz después de 1846, hasta fines del siglo XIX. Quienes estuvieran interesados en ver con un poco más de profundidad la relación entre este nuevo contexto económico que tuvieron que enfrentar los productores estadounidenses, y el nacimiento y desarrollo de la agricultura científica, pueden consultar así mismo nuestro trabajo sobre W.J. Spillman (Serrano-Bosquet y Rivas-Sada 2018). Cabe destacar tan sólo la sustitución de arados de madera por otros de hierro fundido. Ello se debió, sobre todo, a la difusión que sobre nuevos instrumentos llevaron a cabo las sociedades agrícolas que por entonces se estaban constituyendo (Bidwell 1921, 687).

9 La introducción del trigo en Georgia no se dio hasta el siglo XVIII, recordar que la fundación de dicha colonia no se dio hasta 1732 (Ball 1930, 51).

${ }^{10}$ Ejemplo de este descuido lo encontramos en la poca atención pública que recibió, durante este periodo, la protección de la cosecha de trigo frente a enfermedades. Sobre todo si lo comparamos con la promulgación en Francia de las leyes en Rouen, unas leyes que obligaron a erradicación el agracejo europeo con el fin de reducir el aumento de los daños causados por la roya del tallo. Es muy posible que la abundante inmigración francesa, alentada por el gobierno inglés durante esos años, llevara parte de la experiencia e información generada a raíz de la aplicación de dicha ley a los agricultores americanos. Ahora bien, tal y como señala Ball (1930, 51-52), no hubo ninguna acción legislativa al respecto durante los siguientes cien años.

${ }^{11}$ Los primeros experimentos de agricultura científica llevados a cabo en la costa noroeste del Pacífico fueron llevados a cabo por Pedro Alberni entre 1789 y 1794 en el establecimiento San Lorenzo de Nutka (Cutter 1987)

12 El trigo se cultivó por primera vez en las Indias Occidentales en 1521, no como dice Ball en "La historia del mejoramiento del trigo estadounidense" en Columbus en 1494. Autores como Mariano Cárcer (1953) y Justo L. del Río y Lorenzo E. López Reclamo (1996) señalan -basándose en los trabajos de López de Gómara, Bernardino de Sahagún, Francisco Hernández y la Probanza de Juan Garrido- de que fue el liberto negro Juan Garrido quien, por orden de Hernán Cortés, sembró trigo por primera vez en el nuevo continente en 1521.

13 Para obtener más información sobre la producción y la expansión territorial del trigo en la Nueva España desde una perspectiva económica y política, puede consultarse C. E. Suárez (1985). 
${ }^{14}$ Habría que añadir también el ímpetu que pusieron en ello las más altas autoridades que, como el mismo Hernán Cortes y su sucesor en la gobernación, el tesorero Alonso de Estrada, vieron en la comercialización (y monopolización) del pan un gran negocio (Del Río Moreno y López y Sebastián 1996, 35)

15 Recordemos que para entonces (1770) la actual California, Nevada, Utah y Arizona y buena parte de Nuevo México, formaban parte de Nueva España.

${ }^{16}$ Algunas de estas variedades eran todavía ampliamente cultivadas en la costa del Pacífico a inicios del siglo XX. (Ball 1930, 50).

17 Por regla general los trigos denominados Club (Triticum compactum) suelen distinguirse de los trigos comunes (T. vulgare) por sus picos claramente compactos o densos, puntas más cortas y más densas comprimidas lateralmente y tallos generalmente rígido y fuertes. Las plantas de trigo Club pueden ser de cualquiera de invierno o primavera y el tallo puede ser alto o bajo (Bayles y Clark 1954, 147).

18 Introducido por los españoles el Little Club era un trigo con una caña corta y fuerte que no se doblaba y aplanaba en el suelo durante las tormentas de viento y con cabezas apretadas que no permitían que se dispersaran las semillas durante la cosecha (Carlson 2005, 57).

19 Una excelente descripción de la transformación gradual de los métodos de producción en Nueva Inglaterra, desde sus inicios coloniales hasta la primera ola de producción en masa, se puede encontrar en el trabajo de Dirk J. Struik (1991). Destacan los capítulos 1 "La construcción colonial" (25-66) y 5 "Los comienzos de la producción en masa" (175-185).

${ }^{20}$ Ejemplo de ello son Washington y Jefferson. Véase True $(1937,5-6)$

21 Una fanega equivale a 55,5 litros.

22 Ley que creada por Abraham Lincoln el 20 de mayo de 1862 a partir de la cual, cualquier persona que nunca hubiese tomado las armas contra el gobierno de los EE. UU., podía solicitar la titularidad de una propiedad de 65 hectáreas que hubiera cultivado durante cinco años.

${ }^{23}$ Véase en ese sentido los artículos de O.A. Willard (1857) y Timothy K. Minella (2016, 435436).

${ }^{24}$ Segunda sociedad agrícola más antigua de los Estados Unidos, tras la South Carolina Society for Promotion and Improving Agriculture and Other Rural Concerns proyectada en 1784 (True 1937, 6-7).

${ }^{25}$ La resistencia de muchos grupos de campesinos es comprensible a la luz de los intereses políticos (secesionistas, estatales, regionales, etc.) y económicos (sociales y personales) que rodearon muchos de los intentos de introducción de nuevas variedades agrícolas y ganaderas, así como de conocimientos teóricos y prácticos de Europa. Quienes estuvieran interesados en ver un ejemplo de ello se les recomienda revisar el texto de Chester McArthur Destler (1968).

${ }^{26}$ Recordemos y tengamos en cuenta que estas tierras nunca antes habían sido cultivadas. La expansión de la producción de trigo a estos territorios fue también un proceso de agriculturalización.

${ }^{27}$ Este trigo duro, que se hizo popular a principios de siglo, era una especie diferente del trigo común no solo desde una perspectiva biológica: su calidad y las propiedades de su harina lo convertieron en una variedad a considerar desde el punto de vista comercial.

${ }^{28}$ Quienes deseen saber a detalle las diferentes variedades de trigo que fueron introducidas en esta región en el siglo XIX, pueden consultar el texto de Shepherd (1980, 54). Ahí encontrarán 
una excelente lista de dichas variedades así como bibliografía que permita completar esta visión. En el trabajo de Carlson podrá encontrar un análisis más profundo sobre los tipos de prácticas y hábitos del cultivo de trigo en la región $(2005,56)$.

29 Muchas de las variedades cultivadas en 1949 en EEUU eran resultado de la hibridación de Little Club con otras variedades. Es el caso, por ejemplo, de las variedades originadas en la Estación Experimental Agrícola de Washington, Pullman: Triplet cruce (Jones Fife y Little Club) X (Jones Fife X Turkey) [ (Bayles y Clark 1954, 106)]; Hybrid 128, cruce de Jones Winter Fife y Little Club (Bayles y Clark 1954, 149) e Hybrid 63, cruce de Turkey y Little Club (Bayles y Clark 1954, 150-151).

${ }^{30}$ Entre las que podemos considerar la mejora arquitectónica de las construcciones agrícolas. Ejemplo de ello lo tenemos en el trabajo de Wilbur Zelinsky sobre los graneros en Nueva Inglaterra (1958).

${ }^{31}$ De hecho, los ferrocarriles habían reclutado agricultores para que se establecieran a lo largo de sus rutas con el fin de crear clientes para el comercio ferroviario.

$32 \mathrm{El}$ acceso que a bibliotecas, manuales científicos, revistas nacionales e internacionales, informes científicos, revistas de asociaciones e informes gubernamentales a los que, entre otros documentos, tenían a su disposición en las estaciones experimentales es un ejemplo de ello. Véase en ese sentido (True 1937, 140).

${ }^{33}$ En el trabajo de Alfred Charles True (1937) hay una excelente relación y estudio tanto de estas instituciones de investigación agrícola como de algunos de los más importantes o representativos proyectos llevados a cabo en los mismos.

${ }^{34}$ Si bien el artículo en el que se dio a conocer su trabajo apareció en 1902, los trabajos fueron llevados a cabo -tal y como demostramos en un artículo recientemente publicado sobre los mismos (Serrano-Bosquet y Rivas-Sada 2018)- durante la década previa.

${ }^{35}$ Charles Chamberlain Hurst publicó en un artículo en 1903 en Journal of the Royal Horticultural Society of London el que, bajo el título "Mendel's Principles Applied to Wheat Hybrids" reproducía íntegramente el texto de Spillman (1902a) y mostraba cómo el trabajo del agrónomo estadounidense constituía una confirmación independiente de los principios de Mendel.

36 Un ejemplo de ello lo tenemos en el hecho de que la mayoría de los trabajos realizado en las estaciones experimentales en el siglo XX todavía se basaran en la producción y compra de grandes colecciones de muestras, así como en las colecciones generales de las universidades con concesiones de tierras, a menudo, más grandes y extensas del país (True 1937, 139).

Francisco Javier Serrano-Bosquet es Doctor en Filosofía por la Universidad Complutente de Madrid, Profesor asociado del Departamento de Estudios Humanísticos, Escuela de Humanidades y Educación del Tecnologico de Monterrey, Monterrey, Nuevo León, México. Correo electrónico: fjavierserrano@tec.mx. ORCID: https://orcid.org/0000-0003-3929-4141. 